\title{
Research Paper: Augmenting Peripheral Nerve Regeneration Using Hair Follicle Stem Cells in Rats
}

\author{
Leila Beigom Hejazian' ${ }^{\text {(D), Zeinab Akbarnejad }}{ }^{2}$ (D), Fatemeh Moghani Ghoroghi ${ }^{3}$ (D), Banafshe Esmaeilzade ${ }^{4 *}$ (iD, Samira Chaibakhsh ${ }^{5}$ (i)
}

1. Department of Anatomy, School of Medicine, Babol University of Medical Sciences, Babol, Iran

2. ENT and Head \& Neck Research Center, The five Senses Health Institute, School of Medicine, Iran University of Medical Sciences, Tehran, Iran.

3. Department of Anatomy, School of Medicine, Tehran University of Medical Sciences, Tehran, Iran.

4. Department of Anatomy, School of Medicine, Bushehr University of Medical Sciences, Bushehr, Iran.

5. Eye Research Center, the Five Senses Institute, Rasoul Akram Hospital, Iran University of Medical Sciences, Tehran, Iran.

\begin{tabular}{|l|l|l}
$\begin{array}{c}\text { use vour device to scan } \\
\text { and read the article online }\end{array}$ & $\begin{array}{l}\text { Crtation Hejazian, L. B, Akbarnejad, Z., Moghani Ghoroghi, F., Esmaeilzade, B., Chaibakhsh, S. (2021). Augmenting Periph- } \\
\text { eral Nerve Regeneration Using Hair Follicle Stem Cells in Rats. Basic and Clinical Neuroscience, 13(1), 57-70. http://dx.doi. } \\
\text { org/10.32598/bcn.2021.2240.1 }\end{array}$ \\
doi http://dx.doi.org/10.32598/bcn.2021.2240.1
\end{tabular}

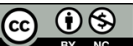

Article info:

Received: 19 Feb 2019

First Revision: 23 Feb 2020

Accepted: 01 Aug 2020

Available Online: 01 Jan 2022

Keywords:

Peripheral nerve regeneration, Rat hair follicle stem cell, Sciatic nerve

\begin{abstract}
$\underline{\text { AB S T RACT }}$
Introduction: Cell therapy is the most advanced treatment of peripheral nerve injury. This study aimed to determine the effects of transplantation of hair follicle stem cells on the regeneration of the sciatic nerve injury in rats.

Methods: The bulge region of the rat whisker were isolated and cultured. Morphological and biological features of the cultured bulge cells were observed by light microscopy and immunocytochemistry methods. Percentages of CD34, K15, and nestin cell markers expression were demonstrated by flow cytometry. Rats were randomly divided into 3 groups of injury, epineurium, and epineurium with cells in which rat Hair Follicular Stem Cells (rHFSCs) were injected into the site of the nerve cut. HFSCs were labeled with Bromodeoxyuridine (BrdU), and double-labeling immunofluorescence was performed to study the survival and differentiation of the grafted cells. After 8 weeks, electrophysiological, histological, and immunocytochemical analysis assessments were performed.

Results: Rat hair follicle stem cells are suitable for cell culture, proliferation, and differentiation. The results suggest that transplantation of rat hair follicle stem cells can regenerate sciatic nerve injury; moreover, electrophysiology and histology examinations show that sciatic nerve repair was more effective in the epineurium with cell group than in the other experimental group $(\mathrm{P}<0.05)$.

Conclusion: The achieved results propose that hair follicle stem cells improve axonal growth and functional recovery after peripheral nerve injury.
\end{abstract}

\section{* Corresponding Author:}




\section{Highlights}

- This study showed that rat hair follicle stem cells are suitable for cell culture, proliferation and differentiation

- The results suggested that transplantation of rat hair follicle stem cells had the potential capability of regenerating sciatic nerve injury

- Evidence of electrophysiology and histology showed Concomitant use of epineurium with hair follicle stem cell was more effective repairment.

\section{Plain Language Summary}

Although repairing damaged peripheral nerves has always been a medical challenge, but peripheral nerve injury has been successfully repaired using various procedures such as nerve auto-graft or stem cell therapy. The functional reconstruction is the most important after therapy because of that primary nerve repair or use of nerve autograft, are still accepted as golden standard methods for treatment. Considerable recent interest has been focused on adult stem cells for both research and clinical applications. A highly promising source of relatively abundant and accessible, active, multipotent adult stem cells are obtained from hair follicles. In research the hair follicle stem cells implanted into the gap region of a severed sciatic nerve injury greatly enhanced the rate of nerve regeneration and the restoration of nerve function. Time is one of the several aspects require specific attention in the clinical treatment of peripheral nerve injury. Because delay of nerve injury treatment may cause neurobiological alterations in neurons and Schwann cells, impairing nerve functional recovery and affect neuron survival. In this study, concluded that stem cell injection 2 weeks after injury in the damaged nerve epineurium repairs nerve fibers, while electrophysiology of the leg muscles showed that muscle function was significantly improved. It indicates the repair of muscular innervation and nerve repair. The results pave the way for further research on this topic.

\section{Introduction}

eripheral nerve regeneration is an important clinical problem. The Peripheral Nerve System (PNS) can regenerate nerve cells, and peripheral nerve injury can be successfully repaired using various procedures such as nerve auto-graft (Belkas, Shoichet, \& Midha, 2004). One of the effective methods of repairing peripheral nerve injury is suturing the two ends of the nerve when the resulting gap is short (Millesi, 1984). Axonal regeneration in a peripheral nerve injury needs extrinsic factors that promote growth and supply guidance to the target. To overcome these problems, various cells have been used to facilitate transplantation. The aims of cellular transplantation include bridging the gap, providing a suitable environment to induce axonal regeneration, and promoting neovascularization (Li, Guo, Ahn, Kim, \& Kim, 2014). Different procedures have been applied to improve the regeneration of peripheral nerves; one is seeding cells into the nerve fragments (Belkas et al., 2004; Li et al., 2014; Ishikawa, Suzuki, Dezawa, Kataoka, Ohta, Cho, \& Ide, 2009; Fan, Crawford, \& Xiao, 2011; Dezawa, 2005). The overarching goal of any stem cell-based approach to peripheral nerve injury is to establish a more favorable environment for regenerating axons and, perhaps more importantly, maintain this support for an extended period (Fairbairn, Meppelink, Ng-Glazier, Randolph, \& Winograd, 2015).

Considerable interest has recently been focused on adult stem cells for research and clinical applications. Such cells can circumvent some problems associated with embryonic stem cells, such as immunologic incompatibility. However, most adult stem cells are relatively sparse in indeterminate locations and growth states (Amoh et al., 2005a). A highly promising source of relatively abundant, accessible, active, and multipotent adult stem cells can be obtained from hair follicles.

Hair follicle stem cells, located in the hair follicle bulge, possess stem cell characteristics: multipotency, high proliferative potential, and the ability to enter quiescence (Nobakht et al., 2011). Nestin, a protein marker for neural stem cells, is also expressed in follicle stem cells and their immediate differentiated progeny (Hejazian et al., 2012; Esmaeilzade et al., 2012). Nestin expression in hair follicle stem cells can differentiate between 
cell lines such as neurons, glial cells, keratinocytes, and smooth muscle cells (Amoh et al., 2005a).

Hair follicle stem cells responded to neuregulin-1 and bone morphogenetic protein 2 by generating Schwann cells and chondrocytes, respectively (Sieber-Blum, Grim, Hu, \& Szeder, 2004). In vivo studies show that Nestin-driven hair follicle stem cells can differentiate blood vessels and neural tissues (Amoh et al., 2005a). Hair follicle stem cells can improve vascularization leading to injured tissue repair (Aki, Amoh, Katsuoka, \& Hoffman, 2010; Amoh, Li, L., Yang, Moossa, Katsuoka, Penman, \& Hoffman, 2004).

The hair follicle stem cells implanted into the gap region of a severed sciatic nerve greatly enhanced the rate of nerve regeneration and the restoration of nerve function. Follicle stem cells are primarily transdifferentiated into Schwann Cells (SCs) which are known to support neuron regrowth.

Thus, nestin-positive hair follicle stem cells can promote the regeneration of peripheral nerve injury. These cells provide an important, accessible, and autologous source of adult stem cells for regenerative medicine (Hoffman, 2006; Amoh et al., 2009a; Amoh et al., 2012; Lin, Liu, Zhang, Zhang, Guo, Ren, \& Kong, 2009). Recently, Bhangra reported several studies on using stem cells for peripheral nerve repair in a review article (Bhangra, Busuttil, Phillips, \& Rahim, 2016). None of the previous studies have used rat hair follicle stem cells. While in our previous studies (Nobakht et al., 2011; Hejazian et al., 2012; Esmaeilzade et al., 2012; Esmaeilzade et al., 2014), we could extract, culture, proliferate and differentiate rat Hair Follicular Stem Cells (HFSCs) in vitro and in vivo. Regarding utilization of cell therapy in peripheral nerve injury, our study differs widely from previous studies, which were referred to, especially Faroni, Mobasseri, S. Kingham, \& Reid (2015) and Bhangra et al. (2016) studies.

In several previous studies, the stem cells extracted from hair follicles are different in type; in other words, several types of stem cells could be found in the bulge area, for instance, the origin of stem cells in neural crest stem cells in the follicle of hair which show different markers (Lin,et al., 2009; Hoffman, 2006). Also, the animal sample used is another difference (transgenic mice with fluorescent protein GFP) (Amoh 2009b; Amoh et al., 2012).

Unlike others, in our study, there were neither other interventions, e.g. nerve transplantation and neurotrophin, nor other cells like the Schwann cell. Consequently, in the current work, in repairing peripheral nerve injuries without any medium, the potential of hair follicle cells was solely evaluated via nestin and CD34 markers, and modified methods were used compared to other studies.

\section{Methods}

\section{Animals and housing conditions}

Forty male Wistar rats (250-300 g body weight) were purchased from the Animal Center of Tehran University of Medical Sciences. All animal experiments were carried out according to the guidelines of the Iranian Council for Use and Care of Animals, approved by the Animal Research Ethics Committee of Iran University of Medical Sciences (Tehran, Iran). All rats were maintained in a temperature-controlled environment of $24 \pm 1^{\circ} \mathrm{C}$ with a $12 \mathrm{~h}$ dark/light cycle (Dark cycle: 8:00 PM to 8:00 AM) with free access to water and food.

The rats were divided into four groups $(n=10$ in each group). First, skin incisions and muscles were retracted to expose the sciatic nerves in all rats. In the sham group, the nerve has been released a little and returns to its original condition. In the injury group, a $10-\mathrm{mm}$ piece was removed from the middle part of the sciatic nerve. In the epineurium group, the nerve was cut and then sutured. In the epineurium plus cell group, HFSCs were injected into the region.

\section{Hair follicle isolation and cultivation}

Albino Wistar rats ( $\mathrm{n}=10$, weighing 250-300 g, 8-10 weeks old, purchased from the Animal Center of Tehran Medical University, Tehran, Iran) were used. The rats were sacrificed with ether, and their whisker follicles were dissected as described by Sieber-Blum and Grim (Sieber-Blum \& Grim, 2004). The tissues were trimmed into small pieces $\left(4 \times 8 \mathrm{~mm}^{2}\right)$, and the samples were incubated in $2 \mathrm{mg} / \mathrm{mL}$ collagenase I/dispase II solution (Sigma-Aldrich, USA). Most of the connective tissues and dermis around the follicles were removed, and the whisker follicles lifted out. The bulge region was then amputated from the upper follicle by making two transversal cuts at the site of enlargement spots of the outer root sheath with a fine needle. The culture procedure was performed with a slight modification, as previously described by Yang and colleagues (Yang et al., 2005). Briefly, 20 isolated bulges were cut into small pieces, plated into flask culture plates, pre-coated with collagen type I (Sigma-Aldrich, USA), and immersed in a 3:1 Dulbecco's MEM (DMEM): nutrient mixture F-12 supplemented with the epidermal growth factor containing 
$10 \%$ fetal bovine serum. All dissection and cultivation procedures were performed under sterile conditions, and incubation was at $37^{\circ} \mathrm{C}\left(5 \% \mathrm{CO}_{2}\right)$. Within approximately 4 days, the initiation of outgrowth of bulge cells from the bulges was observed (Figure 1 A, B). One week after the onset of the outgrowth, the bulges were removed from the culture plates, and the cells were collected by incubation with a mixture $(1: 1)$ of $0.125 \%$ trypsin (Sigma-Aldrich, USA) and $0.02 \%$ EDTA (Sigma-Aldrich, USA) at $37^{\circ} \mathrm{C}$ for 2 minutes. The dispersed cells were centrifuged at $259 \times \mathrm{g}$ for 10 minutes, placed in other collagen-coated plates, and incubated for 3 days with a medium. After sufficient confluence, the cells were detached by trypsin and counted by a Neobar lam.

\section{Flow cytometry}

Hair follicle stem cells isolated from the bulge region were incubated with every $100 \mathrm{~mL}$ primary antibody, mouse anti-CD34 monoclonal antibody (1:75, Sigma, USA), and mouse anti-nestin monoclonal antibody (1:200, Millipore, USA) at room temperature for $1 \mathrm{~h}$. The cells were centrifuged with 1-2 mL of PhosphateBuffered Saline (PBS) (0.1 M).

Subsequently, the cells were incubated in the dark at room temperature for $1 \mathrm{~h}$ following conjugate secondary antibody: goat anti-mouse FITC-conjugate $\operatorname{IgG}(1: 1400$, Abcam, UK). After this time, the percentage of CD34+, $\mathrm{K} 15$, and nestin+were analyzed by flow cytometry.

\section{Immunocytochemistry}

Cells seeded on collagen-coated coverslips $\left(1 \times 10^{5}\right.$ cells per $\mathrm{mL}$ ) were washed 3 times with PBS for 5 minutes and fixed in $4 \%$ paraformaldehyde for 10 minutes. The fixed cells were then washed with PBS for $3 \times 5 \mathrm{~min}$ and incubated in a blocking buffer $(10 \%$ goat serum [Invitrogen, USA] $0.3 \%$ Triton X-100 [Fluka, USA]) at room temperature for 30 minutes. They were then incubated at $4{ }^{\circ} \mathrm{C}$ overnight with the following primary antibody mouse anti-nestin monoclonal antibody (1:200, Millipore, USA). The next day, the cells were rinsed for $3 \times 5$ min to remove unbound primary antibodies. Subsequently, they were incubated at room temperature for 2 hours with the following conjugate secondary antibody: goat anti-mouse FITC-conjugate IgG (1:1400, Abcam, $\mathrm{UK})$. The cell nuclei were counterstained with $1 \mu \mathrm{g} / \mathrm{mL}$ 4, 6-diamidino-2-phenylindole (Sigma-Aldrich, USA) in PBS in the dark at room temperature for $1 \mathrm{~min}$. After washing, the samples were mounted on a slide with mounting media for visualization using a fluorescence microscope. To examine the specificity of the nestin antibody, 3T3 fibroblast-like cells (Pasteur Institute of Iran, Tehran) were used as a negative control. The labeled cells were identified using fluorescent microscopy (Olympus Ax70).

\section{Bromodeoxyuridine (BrdU) labeling}

About 48 to 72 hours before cell transplantation, BrdU (5 $\mu \mathrm{mol} / \mathrm{mL}$; Sigma-Aldrich, St. Louis, MO, USA) was added to the flask of cultured cells. For checking cell labeling with BrdU, $48 \mathrm{~h}$ after cell labeling, the labeled cells on collagen-coated coverslips were washed in PBS for $3 \times 5 \mathrm{~min}$ and fixed in $4 \%$ PFA for $10 \mathrm{~min}$. Then, the fixed cells were washed in PBS for $3 \times 5$ min and incubated in $2 \mathrm{~N} \mathrm{HCl}$ at $60^{\circ} \mathrm{C}$ for $45 \mathrm{~min}$, and were washed 2 times in $0.1 \mathrm{M}$ borate buffer ( $\mathrm{pH} 8.3$ ). After being washed in blocking buffer $(10 \%$ goat serum, Sigma-Aldrich, USA/0.3\% Triton X-100 Fluka, USA and $1 \% \mathrm{BSA}$ ) at room temperature for $60 \mathrm{~min}$, the incubated cells were again incubated with the primary antibody anti-BrdU (1:500, Sigma-Aldrich, USA) at $4^{\circ} \mathrm{C}$ overnight. The next day, the cells were rinsed in PBS for $3 \times 5 \mathrm{~min}$ to remove unbound primary antibodies. Subsequently, they were incubated at room temperature for $1 \mathrm{~h}$ with the secondary antibody of goat anti-mouse FITC conjugate IgG (1:200, Abcam, Cambridge, UK), washed in PBS for $3 \times 10 \mathrm{~min}$, mounted with mounting media, and visualized using a fluorescence microscope (Figure 1C).

\section{Transplantation procedure}

The rats were anesthetized by intraperitoneal injection of a combination of ketamine $(100 \mathrm{mg} / \mathrm{kg})$ and xylazine $(10 \mathrm{mg} / \mathrm{kg})$. After the skin incision, the sciatic nerve was exposed using a muscle-splitting incision. Under an operating microscope, the left sciatic nerve was exposed to the mid-thigh, cut, and then sutured with 8-0 propylene sutures in the epineurium group. Also, in the epineurium plus cell group, $5 \times 10^{5}$ cells $/ 50 \mu \mathrm{L}$ were injected into the area. Finally, the skin was sutured.

\section{Histological examination}

Eight weeks after the implantation, the rats were anesthetized and sacrificed, and the regenerated nerves were harvested. The nerves were immediately fixed in a cold buffered $2.5 \%$ glutaraldehyde solution. After fixation, these nerve tissues were post-fixed in $1 \%$ osmium tetroxide, dehydrated, and embedded in resin. The semithin sections were then stained with $1 \%$ toluidine blue. All nerve sections were observed under a light microscope, and photographs were taken using a digital camera. Images of the histological sections were digitized 

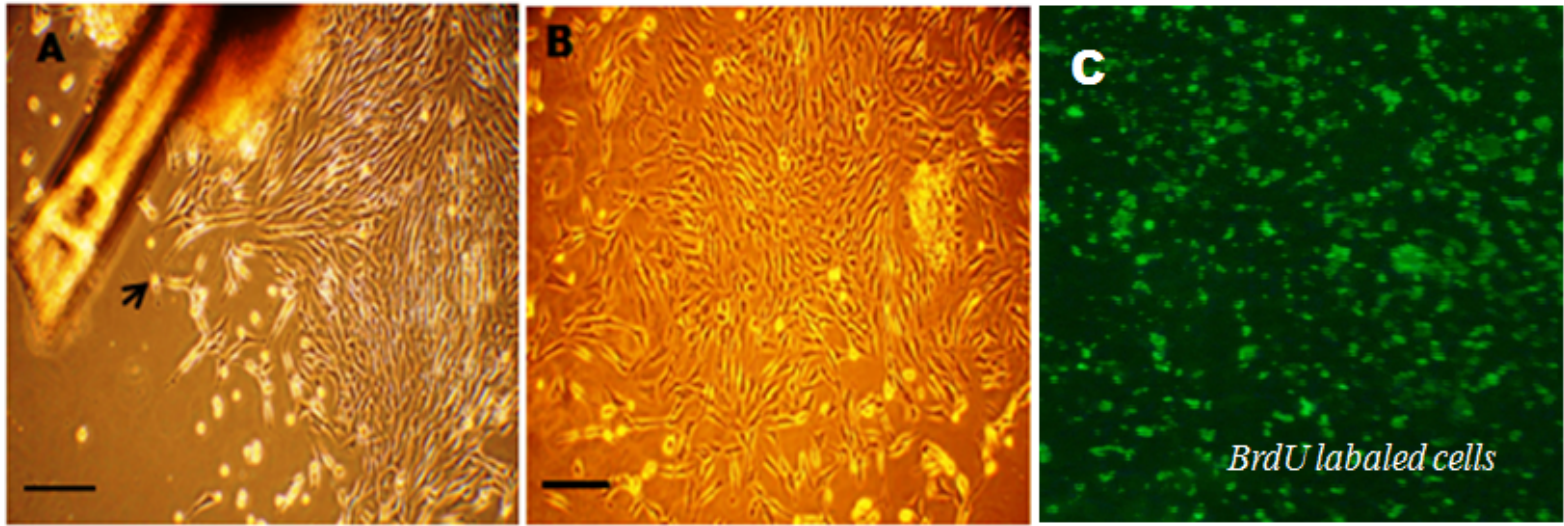

Figure 1. Characteristics of hair follicle stem cells

NEUR

The primary culture of bulge cells from rat hair follicles: A) the growth of bulge cells after 4 days and B) after 7 days. In (C), the hair follicle stem cells were labeled with BrdU 72 hours prior to transplantation (Scale bars $=50 \mu \mathrm{m}$ in A and B).

and subsequently analyzed using an image analyzer system (OLYSIA BioReport). The number of axons was counted in randomly selected fields $\left(4 \times 10^{4} \mu \mathrm{m}^{2}\right)$ at a magnification of $400 \mathrm{X}$.

\section{Immunohistochemistry}

After 8 weeks of cell transplantation for the group of epineurium with cells, the sciatic nerve specimens were put in the medium of $10 \%$ formalin and inserted in paraffin as routine processing; subsequently, 5- $\mu \mathrm{m}$ nerve pieces were accumulated via a rotary microtome (Leitz, 1512, Germany).

Paraffin sections were stained with Envision G12 double-stain system kit (DAKO, USA) according to the kit protocol. The following primary antibodies were used in this protocol: polyclonal anti-S100 antibody (1:400, Dako, USA) and monoclonal anti-BrdU antibody (1:500, Sigma-Aldrich, USA). The secondary antibodies in this protocol were horseradish peroxidase and alkaline phosphatase, that was detected by Diaminobenzidine (DAB) and permanent red staining.

\section{Electrophysiological measurements}

Eight weeks after the transplantation, the rats were anesthetized intraperitoneally with ketamine $(100 \mathrm{mg} /$ $\mathrm{kg}$ ) and xylazine $(10 \mathrm{mg} / \mathrm{kg})$, and the sciatic nerves were exposed. Electric stimulation (duration of $0.1 \mathrm{~ms}$, the intensity of $2.3 \mathrm{~mA}$ ) was applied to the proximal side of the injured nerve. The compound muscle action potential (electromyography) into the belly of the gastrocnemius muscle was recorded in the gastrocnemius with a needle electrode and a reference cap electrode inserted into the knee joint. A stainless steel needle used as the ground electrode was inserted into the tail skin. The area of the recorded muscle response $(\mathrm{mV} \times \mathrm{ms})$ and the amplitude was calculated as these variables can reflect the amount of activated fibers (Chen et al., 2007; Mimura, Dezawa, Kanno, Sawada, \& Yamamoto, 2004).

\section{Statistical analysis}

Data analysis was done using SPSS v 16 software (SPSS, Chicago, IL, USA). All data were analyzed by utilizing the Kruskal-Wallis test. When statistical significance was found between groups, the Mann-Whitney tests were performed to determine significant pairwise differences. The data were expressed as Mean \pm SD, and $\mathrm{P}<0.05$ was considered statistically significant.

\section{Results}

The Primary Culture of Bulge Cells From Rat Hair Follicles

Cells were collected from the bulge area. After 4 days of relocating, the bulges were pulled out, adherent cells were disconnected using trypsin treatment, and finally subcultured. Also, 72 hours before transplantation, the hair follicle stem cells were tagged by BrdU (Figure 1).

\section{Percentage and characterization of cultured HFSCs}

Flow cytometry analysis determined the percentage of CD34 and nestin of cell markers expression in cultured bulge cells (Figure 2). Immunocytochemical staining showed the expression of nestin (neural stem cell marker) in bulge cells. Our results showed that the nestin antibody staining bulge cells were seeded on coverslips (Figure 3). 

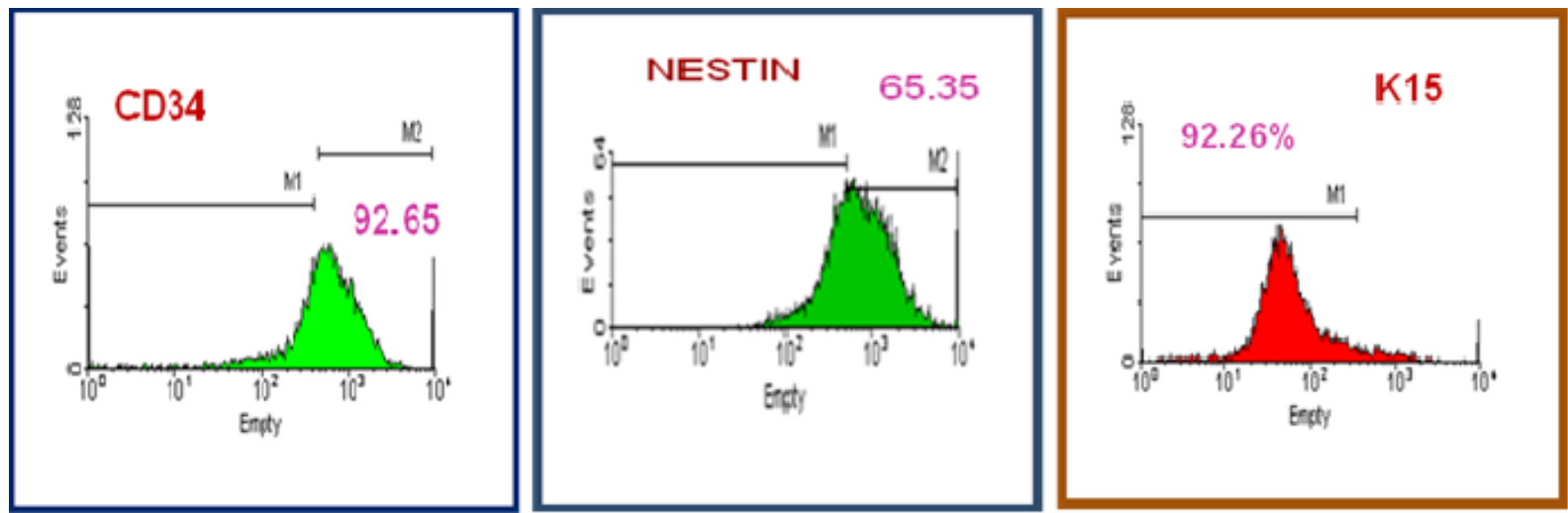

Figure 2. Flow cytometry determining HFSCs primarily stemming cells

Results show the percentage of CD34 and nestin-positive cells. No positive reaction was seen with the K15 antibody. This finding demonstrates that bulge-derived neural stem cells are K15 negative.

Findings of the immunohistochemistry of HFSCs

Using a double-staining procedure, many cells were detected in the transplanted site, which presented $\mathrm{Br}$ dU-S100 and proved that transplanted marked cells differentiated to Schwann cells. Using a double-staining Envision kit (DAKO, USA), we showed that the nuclei of differentiated cells, that had presented BrdU, were brown and presentation of S100 in the cytoplasm was red. The results confirmed the presence, viability, and differentiation of transplanted cells in the severed sciatic nerve 8 weeks after the transplantation (Figure 4).

Electrophysiological results of amplitude and latency

The electrophysiological test results comprised both amplitude and latency measures. The time calibration bar was $2 \mathrm{~ms}$, and the amplitude calibration bar was 10 $\mathrm{mV}$. The stimulation intensity and the duration were 2.3 $\mathrm{mA}$ and $0.1 \mathrm{~ms}$ (Figure 5). Table 1 presents the description and comparison results of amplitude in $\mathrm{mV}$ and latency of the study groups. The pairwise comparisons of groups showed that the mean amplitude was statistically significant in two-by-two comparisons $(0.008<\mathrm{P}<0.010)$ except in the epineurium and the epineurium with cells groups that did not differ significantly. There was a significant difference between groups of amplitude $(\mathrm{P}=0.001)$ and latency $(\mathrm{P}=0.012)$ (Figure 6, 7).

\section{Histological findings}

To evaluate the regeneration efficacy of myelinated and unmyelinated fibers, we examined semi-thin sections of the nerves. Light microscopic examination of toluidine blue-stained cross-sections of these nerves revealed a clear qualitative difference between the experimental and injury groups (Figure 8). Statistical analysis showed a significant difference in myelin thickness of groups $(\mathrm{P}=0.022$, Table 1$)$. The only statistically significant pairwise comparison (Figure 9) was referred to the injury and sham groups $(\mathrm{P}=0.045)$. The nerve fibers' densities differed significantly between groups $(\mathrm{P}=0.027$, Table 1). Pairwise comparisons (Figure 10) showed a
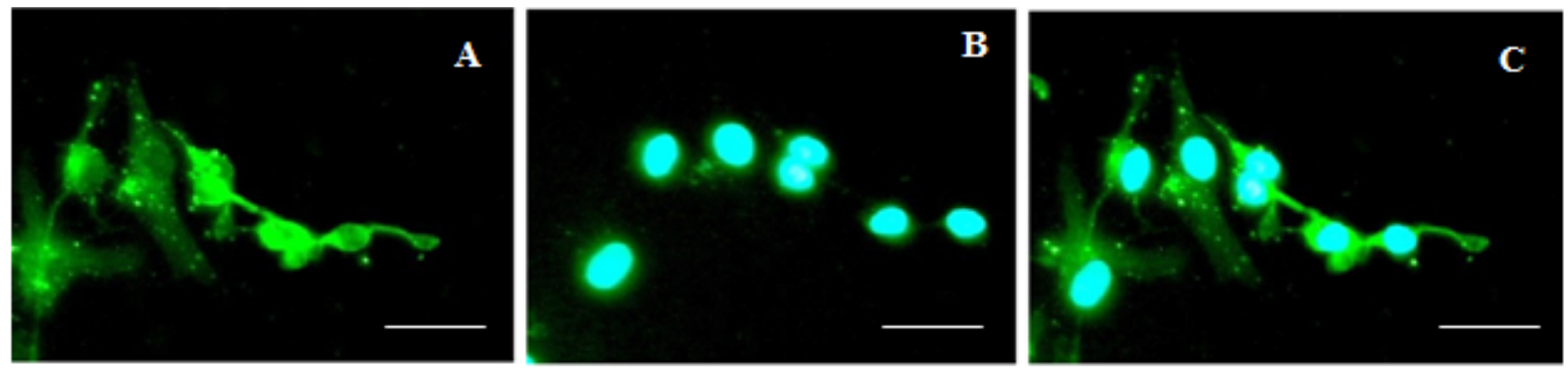

Figure 3. Probing bulge-derived cells with specific antibodies before differentiation

NEUR:SCIENCE

Staining with the nestin antibody and 4,6-diamidino-2-phenylindole (DAPI) nuclear stain represents neural stem cells originating from the neural crest. A) Immunocytochemical cultures of Hair Follicle Stem Cells (HFSCs) were nestin-positive, B) DAPI staining image for nuclei, C) HFSCs were nestin-positive with their nuclei after merge (Scale bars=20 $\mu \mathrm{m}$ in A, B, and C). 


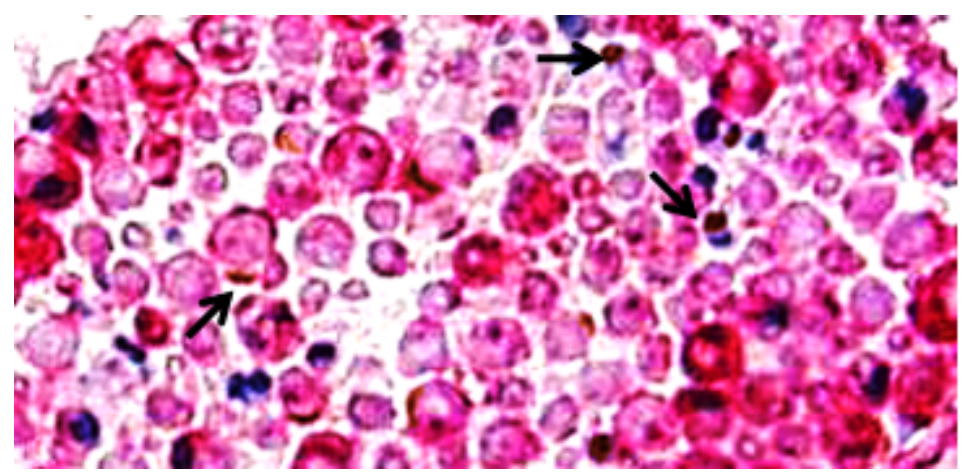

NEUR SCIENCE

Figure 4. Differentiation of Hair Follicle Stem Cells (HFSCs) to schwann cells, 8 weeks after cell transplantation in epineurium with cell group using envision kit

Microscopic immunodetection of S100 (pink) and BrdU (brown) in the site of nerve cut and HFSCs injected in the cells, incubated with bromodeoxyuridine (BrdU) 72 hours before transplantation, then paraffin-embedded sections immunostained with antibodies against BrdU (diaminobenzidine [DAB], brown) and S100 (permanent red, red). (Scale bars $=40 \mu \mathrm{m}$ ).

significant difference between the injury group with others (both $\mathrm{P}=0.045$ ).

\section{Discussion}

Injuries to peripheral nerves are among the most challenging microsurgical problems. These damages are associated with considerable disability due to loss of both motor and sensory functions. There is a need to replace autografts because this procedure entails multiple surgeries, loss of function, and sensation at the donor site (Belkas et al., 2004; Li et al., 2014; Fan et al., 2011).
The great hope in regenerative medicine for nerve repair is the exploitation of the regenerative potential of cell-based therapies. This procedure is of particular relevance, especially for long gaps, where the use of nerve guides alone has failed to provide successful regeneration (Faronia et al., 2015). Nerve regeneration by nerve guidance scaffolds is improved because of less myofibroblast infiltration, accumulation of neurotrophic factors in high concentrations, and reduction of scar formation (Evans, 2001).

Because stem cells are essential seeding cells for peripheral nerve regeneration, special attention has been

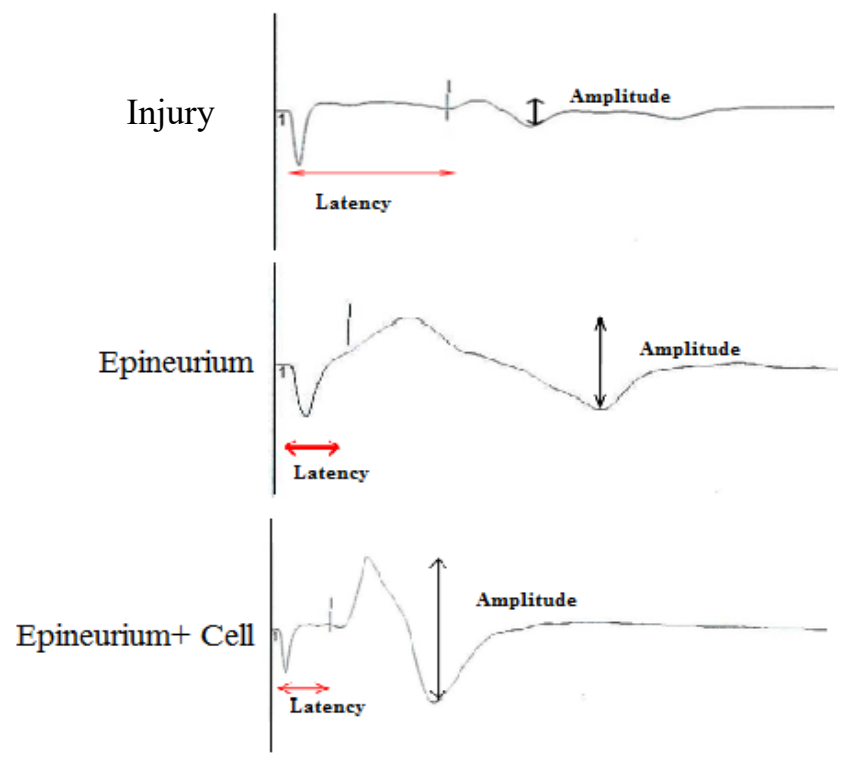

NEUR:SCIENCE

Figure 5. Electrophysiological waves for injury, epineurium, and epineurium with cells groups, 8 weeks after surgery

Amplitude and latency are shown for each group. The time calibration bar was $2 \mathrm{~ms}$, and the amplitude calibration bar was $10 \mathrm{mV}$. The stimulation intensity was $2.3 \mathrm{~mA}$, and the duration was $0.1 \mathrm{~ms}$. 


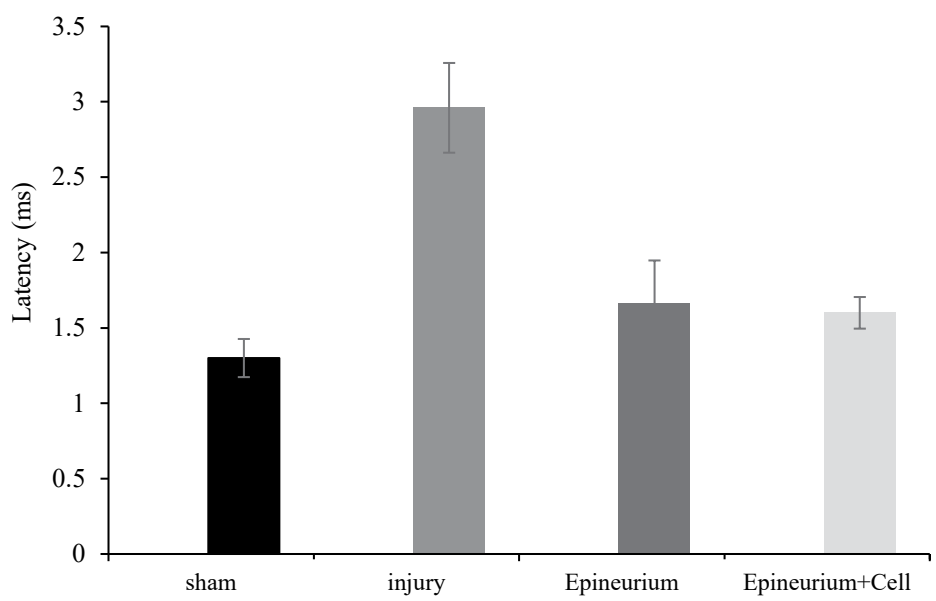

Figure 6. Graphs showing the electrophysiology tests of latency

The results of electrophysiology tests of latency showed statistically significant differences between the injury group and the experimental groups (epineurium and epineurium with cells). Error bars represent Mean $\pm S D(n=10)$.

paid to developing a rich and accessible cellular reservoir for this cell type (Amoh et al., 2005a). In this study, we evaluated the effect of transplantation of rHFSCs on the recovery of rat sciatic nerve injury. Several investigators have shown that HFSCs can repair the injury of mice sciatic nerve (Amoh et al., 2005a; Amoh et al., 2012; Amoh, Hamada, Aki, Kawahara Hoffman, \& Katsuoka, 2010). However, their methods and results were different, and they have not evaluated the results under similar conditions on the rats. Since rats are the most common and accessible laboratory animals, our research is done on this animal. In addition, our team in previous studies succeeded in isolating, culturing, and differentiating these cells in the rat. Recently, other researchers have studied rHFSC characteristics (Quan et al., 2016). Their study has also taken into consideration HFSCs in rats.

Our study confirmed the presence of HFSCs using antinestin antibody and anti-CD34 in vitro. This finding is consistent with previous reports (Hejazian et al., 2012; Amoh et al., 2012).

We cultured and labeled the HFSCs with BrdU. The immunohistochemistry double-stain process showed that BrdU-positive HFSCs can survive in vivo and largely transdifferentiate into SCs (S100 positive cells) at the sciatic nerve injury after 8 weeks.

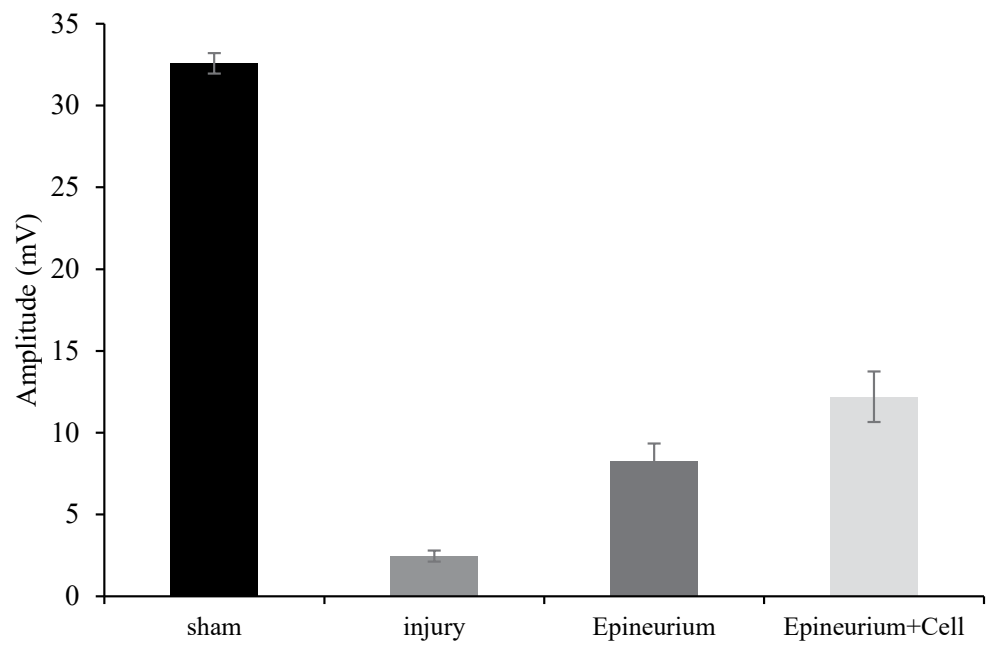

NEUR SCIENCE

Figure 7. Graphs showing the electrophysiology tests of amplitude: the results of electrophysiology tests of amplitude

The amplitude was statistically significant in two-by-two comparisons $(\mathrm{P}<0.05)$. Error bars represent Mean $\pm \mathrm{SD}(\mathrm{n}=10)$. 

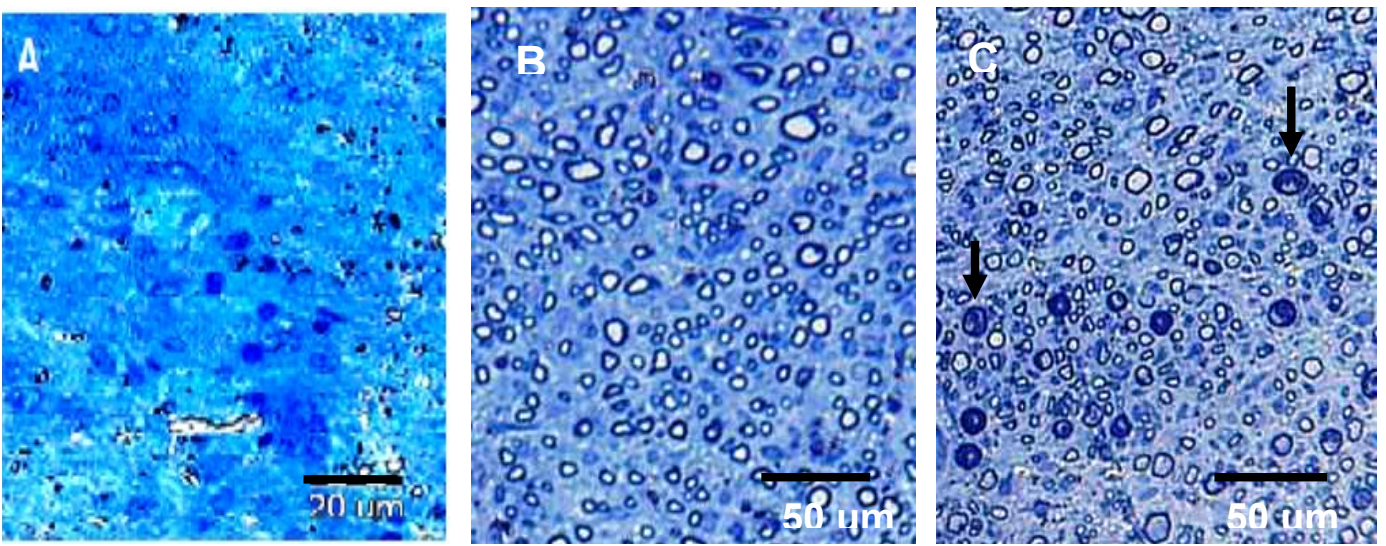

Figure 8. Histology assessment of the sciatic nerve 8 weeks after the operation

NEUR:SCIENCE

The sciatic nerve distal to the nerve transection repair site was harvested. Semi-thin $(500 \mathrm{~nm})$ sections were stained with toluidine blue and were seen by light microscopy: A) Injury B) Epineurium C) Epineurium with cells (arrows show more myelination (Scale bars $=20$ $\mu \mathrm{m}$ in $\mathrm{A}, 50 \mu \mathrm{m}$ in $\mathrm{B}$, and $\mathrm{C}$ ).

During peripheral nerve regeneration, trophic factors and supporting substances are essential molecules (Chen et al., 2000; Lee et al., 2003). Researchers have indicated several mechanisms that may promote functional improvement by HFSCs (Amoh et al., 2005a; Amoh et al., 2012, Amoh et al., 2010).

Hair follicle stem cells were implanted into the gap region of mice's severed sciatic nerve, greatly enhancing the rate of nerve regeneration and the restoration of nerve function. HFSCs can promote axonal regeneration in PNS (Amoh et al., 2005a; Amoh et al., 2010). Neural stem cells are marked by the expression of an intermediated filament of nestin. The expression of the unique protein, nestin, in neural stem cells and hair follicle stem cells suggests their possible relation (Hejazian et al., 2012; Esmaeilzade et al., 2012; Amoh et al., 2005b). These cells mainly transdifferentiate into Schwann cells known to support neuron regrowth (Amoh et al., 2005a; Amoh et al., 2009a; Amoh et al., 2012; Amoh and Hoffman, 2010b). Schwann cells secrete many factors, such as neurotrophic factors that induce tissue plasticity and neuroprotective factors (Rodriguez et al., 2000). SCs can release neurotrophic factors such as NGF, BDNF, GDNF,

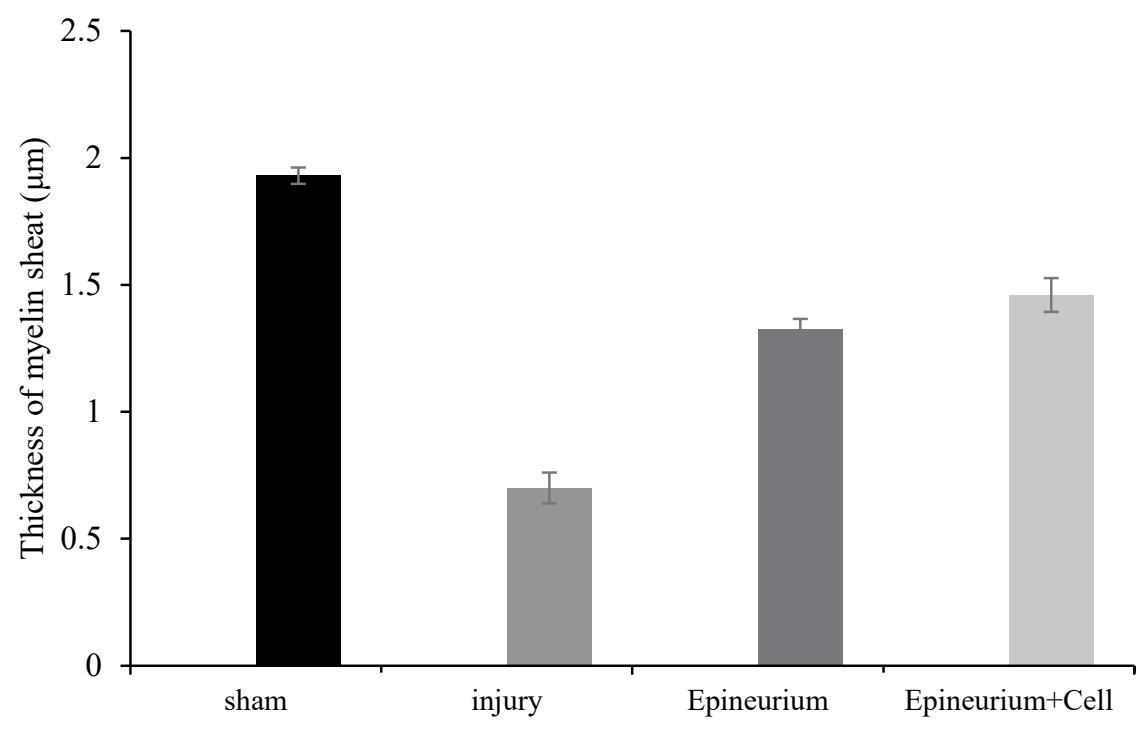

NEUR:SCIENCE

Figure 9. Graphs showing the density of myelinated and unmyelinated fibers; results of histological examination

Statistical analysis showed a significant difference in the myelin thickness of groups $(\mathrm{P}<0.05)$. The only statistically significant pairwise comparison was referred to the injury and sham group. Error bars represent Mean $\pm S D(n=10)$. 


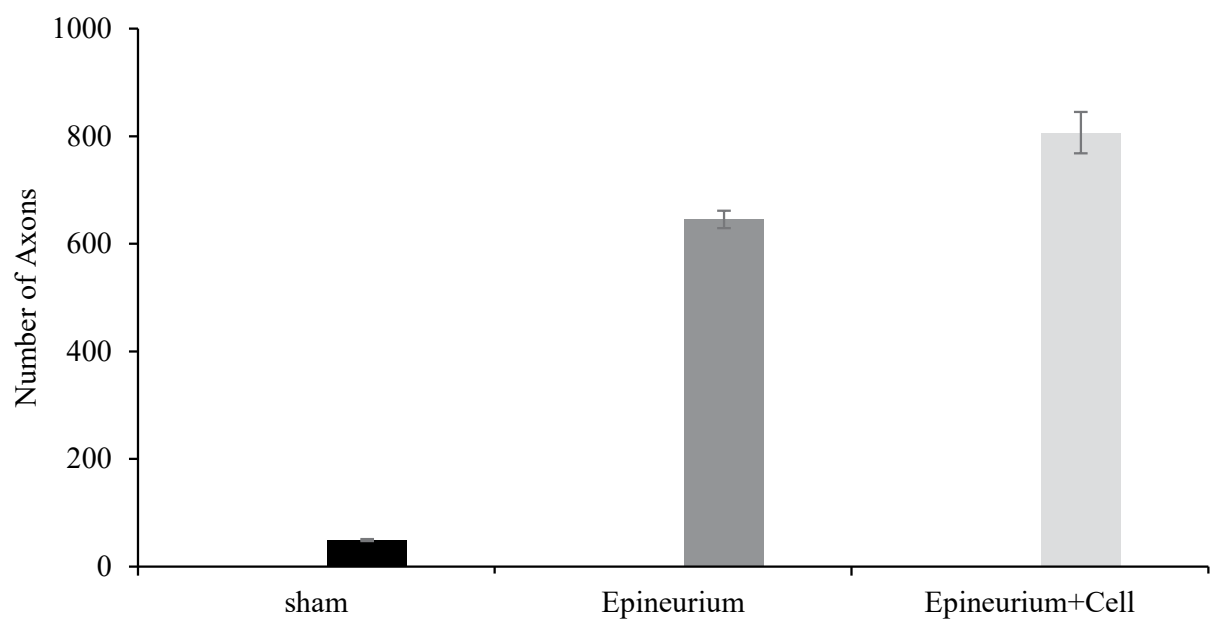

Figure 10. Graphs showing the number of myelinated and unmyelinated fibers

NEUR OSIENCE

The nerve fibers' densities were significantly different between groups. Pairwise comparisons showed a significant difference between the injury group with others $(P<0.05)$. Error bars represent Mean $\pm S D(n=10)$.

CNTF, and VEGF, as well as produce extracellular matrix proteins such as collagen I, collagen IV, fibronectin, and laminin (Moradi et al., 2012; Feneley, Fawcett, \& Keynes, 1991). Therefore, evidence primarily supports the hypothesis that SCs differentiated from transplantdated HFSCs may repair peripheral nerve injuries.

In addition, SCs mediated by immunoglobulin superfamily molecules, like the nerve cell adhesion molecule, protein $0(\mathrm{P} 0)$, cadherin, and protocadherins are essential for axonal elongation and organized sprouting. SCs also produce basal lamina components, like collagen IV and laminin, that play an essential role in nerve regeneration. Among the adhesion molecules, laminin is the most potent factor for promoting axonal outgrowth. It thus seems likely that transplantation of SCs may also repair peripheral nerve injuries (Chew, Mi, Hoke, \& Leong, 2008; Ide, 1996; Walsh, Biernaskie, Kemp, \& Midha, 2009).

Time is one of the several aspects that require specific attention in the clinical treatment of peripheral nerve in- jury. Delay in nerve injury treatment may cause neurobiological alterations in neurons and Schwann cells, impair functional nerve recovery, and affect neuron survival (Egle De Stefano, Toni, D’Orazi, Ortensi, \& Tata, 2013). In this study, we transplanted nestin-expressing cells after two weeks. In another study, cells were cultured for 1-2 months before transplantation to the injured nerve, which would not be optimal for clinical application because the patient should be treated soon after injury.

The condition velocity is a quantitative and valid index for evaluating action potential conduction in peripheral nerves (Ao Q et al., 2011). After the growth of sufficiently regenerated fibers across the nerve bridge, the target muscle can construct muscle action potential, which might be measurable. Additionally, the condition velocity relies directly on factors such as the diameter of axons, the thickness of the myelin sheath, and the length of internodes (Matsumoto K et al., 2000).

Table 1. Comparing amplitude, latency, myelination, and number of axons between four study groups

\begin{tabular}{|c|c|c|c|c|c|c|c|c|c|c|c|c|}
\hline \multirow{2}{*}{ Groups } & \multicolumn{3}{|c|}{ Amplitude } & \multicolumn{3}{|c|}{ Latency } & \multicolumn{3}{|c|}{ Myelin Thickness } & \multicolumn{3}{|c|}{ Number of Axons } \\
\hline & Mean $\pm S D$ & $\chi^{2} / \mathrm{df}$ & $P$ value & Mean \pm SD & $\chi^{2} / \mathrm{df}$ & $\mathbf{P}$ & Mean $\pm S D$ & $\chi^{2} / d f$ & $\mathbf{P}$ & Mean \pm SD & $\chi^{2} / \mathrm{df}$ & $\mathbf{P}$ \\
\hline Sham & $32.58 \pm 1.40$ & & & $1.30 \pm 0.28$ & & & $1.93 \pm 0.06$ & & & $49.33 \pm 3.22$ & & \\
\hline Injury & $2.46 \pm 0.76$ & & & $2.96 \pm 0.67$ & & & $0.70 \pm 0.11$ & & & - & & \\
\hline Epineurium & $7.96 \pm 2.42$ & $16.27 / 3$ & 0.001 & $1.66 \pm 0.64$ & $10.94 / 3$ & 0.012 & $1.33 \pm 0.07$ & $9.63 / 3$ & 0.022 & $645.33 \pm 28.02$ & $7.22 / 2$ & 0.027 \\
\hline Epineurium+Ce & $12.20 \pm 3.45$ & & & $1.60 \pm 0.23$ & & & $1.46 \pm 0.12$ & & & $806.67 \pm 66.58$ & & \\
\hline
\end{tabular}


The magnitude of EMG correlates directly to the number of nerve fibers that innervate the muscle; thus, the conduction velocity of the motor nerve could be calculated.

EMG examinations give a vital index for the function of conduction in the peripheral nerve (Wang, $\mathrm{Hu}, \mathrm{CaO}$, Yao, Wu, \& Gu, 2005). In this paper, the recovery index of EMG magnitude and the medium percentage of conduction velocity illustrated that animals in the epineurium and epineurium with cell groups experience a considerable improvement in nerve regeneration.

The recovery index was related to several factors, including the diameter of the regenerating axons, thinner myelin sheaths with shorter internodes, and immaturity of myelinated nerve fibers as a whole (Matsumoto K et al., 2000; Wang X et al., 2005) that was consistent with morphometric findings.

In this study, we showed the benefit of repairing nerve defects using HFSCs, as evidenced by electromyography tests in the gastrocnemius muscle and histological examination measured by myelinated and unmyelinated axonal number analysis. The results of the histological examination showed statistically significant differences between the injury group and the experimental groups (epineurium and epineurium with cells). Our results showed fewer axons, fewer myelinated fibers, reduced nerve fiber density, increased axonal degeneration, some axonal atrophy, and a reduction of the mean thickness of the myelin sheath in the injury group compared with other groups. The epineurium and the epineurium with cell groups showed an increased number of distinct axons, increased nerve fiber density, increased number of regenerating axons, and reduced axonal degeneration. Some previously reported data support our findings (Amoh et al. 2005a). However, there is no significantly higher thickening of myelinated fibers in the experimental groups.

The number of nerve fibers density was higher in the experimental groups compared to the injury group (Figure 10). However, the axonal regeneration seen in the epineurium with cells group is much greater than in other groups.

The latency diagram shows the delay in the conduction of the electric wave, which indicates the velocity of the conduction of the electric wave in the neuromuscular synapse. So, whatever the level of latency is lower, the function of the synapse is better, and the nerve is repaired better. These findings confirm those documented previously (Fan et al., 2011; Ide, 1996).

\section{Conclusion}

The current work revealed that the injection of hair follicle cells recovers severely injured sciatic nerve, which could develop gastrocnemius muscle via electric stimulation. Eight weeks after transplantation of hair follicle stem cells, the histological evaluation revealed a higher concentration of myelinated and unmyelinated fibers within the nerve in the epineurium with cells group. The results propose that hair follicle stem cells improve axonal growth and functional recovery after peripheral nerve injury.

\section{Ethical Considerations}

\section{Compliance with ethical guidelines}

There were no ethical considerations to be considered in this research.

\section{Funding}

This study was financially supported by grant No. 12311 from Minimally Invasive Surgery Research Center, Rassoul Akram Hospital, and Iran University of Medical Sciences.

\section{Authors' contributions}

Conceptualization, Methodology, Investigation, and Writing - original draft: Leila Beigom Hejazian; Banafshe Esmaeilzade; Data collection and Writing - review \& editing: Zeinab Akbarnejad, Fatemeh Moghani Ghoroghi; Data analysis: Samira Chaibakhsh.

\section{Conflict of interest}

The authors declared no conflict of interest.

\section{Acknowledgments}

We thank the Minimally Invasive Surgery Research Center, Rasoul Akram Hospital, and Iran University of Medical Sciences for their financial support. Also, we would like to acknowledge the Departments of Anatomy and Cellular and Molecular Research Center, School of Medicine, Iran University of Medical Science, Tehran, Iran. 


\section{References}

Aki, R., Amoh, Y., Li, L., Katsuoka, K., \& Hoffman, R. M. (2010) Nestin-expressing interfollicular blood vessel network contributes to skin transplant survival and wound healing Journal of Cellular Biochemistry, 110(1), 80-6. [DOI:10.1002/ jcb.22512] [PMID]

Amoh, Y., \& Hoffman, R. M. (2010b) Isolation and culture of hair follicle pluripotent stem (hfPS) cells and their use for nerve and spinal cord regeneration. Methods in Molecular Biology, 585, 401-20. [DOI:10.1007/978-1-60761-380-0_28] [PMID]

Amoh, Y., Aki, R., Hamada, Y., Niiyama, S., Eshima, K., Kawahara, K., et al. (2012). Nestin-positive hair follicle pluripotent stem cells can promote regeneration of impinged peripheral nerve injury. The Journal of Dermatology, 39(1), 33-8. [DOI:10.1111/j.1346-8138.2011.01413.x] [PMID]

Amoh, Y., Hamada, Y., Aki, R., Kawahara, K., Hoffman, R. M., \& Katsuoka, K. (2010a). Direct transplantation of uncultured hair-follicle pluripotent stem (hfPS) cells promotes the recovery of peripheral nerve injury. Journal of Cellular Biochemistry, 110(1), 272-7. [DOI:10.1002/jcb.22534] [PMID]

Amoh, Y., Kanoh, M., Niiyama, S., Hamada, Y., Kawahara, K., Sato, Y., et al. (2009b). Human hair follicle pluripotent stem (hfPS) cells promote regeneration of peripheral-nerve injury: An advantageous alternative to ES and iPS cells. Journal of Cellular Biochemistry, 107(5), 1016-20. [DOI:10.1002/ jcb.22204] [PMID]

Amoh, Y., Li, L., Campillo, R., Kawahara, K., Katsuoka, K., Penman, S., \& Hoffman, R. M. (2005a). Implanted hair follicle stem cells form Schwann cells that support repair of severed peripheral nerves. Proceedings of the National Academy of Sciences, 102(49), 17734-8. [DOI:10.1073/pnas.0508440102] [PMID] [PMCID]

Amoh, Y., Li, L., Katsuoka, K., \& Hoffman, R. M. (2009a). Multipotent nestin-expressing hair follicle stem cells. The Journal of dermatology, 36(1), 1-9. [DOI:10.1111/j.13468138.2008.00578.x] [PMID]

Amoh, Y., Li, L., Katsuoka, K., Penman, S., \& Hoffman, R. M. (2005b). Multipotent nestin-positive, keratin-negative hairfollicle bulge stem cells can form neurons. Proceedings of the National Academy of Sciences, 102(15), 5530-4. [DOI:10.1073/ pnas.0501263102] [PMID] [PMCID]

Amoh, Y., Li, L., Yang, M., Moossa, A. R., Katsuoka, K., Penman, S., \& Hoffman, R. M. (2004). Nascent blood vessels in the skin arise from nestin-expressing hair-follicle cells. Proceedings of the National Academy of Sciences, 101(36), 13291-5. [DOI:10.1073/pnas.0405250101] [PMID] [PMCID]

Ao, Q., Fung, C. K., Tsui, A. Y. P., Cai, S., Zuo, H. C., Chan, Y. S., et al. (2011). The regeneration of transected sciatic nerves of adult rats using chitosan nerve conduits seeded with bone marrow stromal cell-derived Schwann cells. Biomaterials, 32(3), 787-96. [DOI:10.1016/j.biomaterials.2010.09.046] [PMID]

Belkas, J. S., Shoichet, M. S., \& Midha, R. (2004). Peripheral nerve regeneration through guidance tubes. Neurological Research, 26(2), 151-60. [DOI:10.1179/016164104225013798] [PMID]

Bhangra, K. S., Busuttil, F., Phillips, J. B., \& Rahim, A. A (2016). Using stem cells to grow artificial tissue for peripheral nerve repair. Stem Cells International, 2016, 7502178. [DOI:10.1155/2016/7502178] [PMID] [PMCID]
Chen, C. J., Ou, Y. C., Liao, S. L., Chen, W. Y, Chen, S. Y., Wu, C. W., et al. (2007). Transplantation of bone marrow stromal cells for peripheral nerve repair. Experimental Neurology, 204(1), 443-53. [DOI:10.1016/j.expneurol.2006.12.004] [PMID]

Chen, Y. S., Hsieh, C. L., Tsai, C. C., Chen, T. H., Cheng, W. C., $\mathrm{Hu}, \mathrm{C}$. L., et al. (2000). Peripheral nerve regeneration using silicone rubber chambers filled with collagen, laminin and fibronectin. Biomaterials, 21(15), 1541-7. [DOI:10.1016/S0142 9612(00)00028-4] [PMID]

Chew, S. Y., Mi, R., Hoke, A., \& Leong, K. W. (2008). The effect of the alignment of electrospun fibrous scaffolds on Schwann cell maturation. Biomaterials, 29(6), 653-61. [DOI:10.1016/j. biomaterials.2007.10.025] [PMID] [PMCID]

Dezawa, M. (2005). [Future views and challenges to the peripheral nerve regeneration by cell based therapy (Japanese)] Rinsho Shinkeigaku, 45(11):877-9. [PMID]

Egle De Stefano, M., Toni, F., D'Orazi, V., Ortensi, A., \& Tata A. M. (2013). Therapeutic approaches enhancing peripheral nerve regeneration. Advances in Bioscience and Biotechnology, 4(6A), 53-60. [DOI:10.4236/abb.2013.46A008]

Esmaeilzade, B., Nobakht, M., Hosseini, S. M., Mortazavi, P., Barati, M., Mehrabi, S., et al. (2014). Adult neurogenesis of epidermal neural crest stem cells (EPI-NCSC) in hippocampus of Alzheimer's rat model. Comparative Clinical Pathology, 23(3), 789-97. [DOI:10.1007/s00580-013-1690-z]

Esmaeilzade, B., Nobakht, M., Joghataei, M. T., Roshandel, N. R., Rasouli, H., Kuchaksaraei, A. S., et al. (2012). Delivery of epidermal neural crest stem cells (EPI-NCSC) to hippocamp in Alzheimer's disease rat model. Iranian Biomedical Journal, 16(1), 1-9. [DOI:10.6091/ibj.1029.2012] [PMID] [PMCID]

Evans, G. R. (2001). Peripheral nerve injury: A review and approach to tissue engineered constructs. The Anatomical Record, 263(4), 396-404. [DOI:10.1002/ar.1120] [PMID]

Fairbairn, N. G., Meppelink, A. M., Ng-Glazier, J., Randolph, M A., \& Winograd, J. M. (2015). Augmenting peripheral nerve regeneration using stem cells: A review of current opinion. World Journal of Stem Cells, 7(1), 11-26. [DOI:10.4252/wjsc. v7.i1.11] [PMID] [PMCID]

Fan, W., Crawford, R., \& Xiao, Y. (2011). The ratio of VEGF/ PEDF expression in bone marrow mesenchymal stem cells regulates neovascularization. Differentiation, 81(3), 181-91. [DOI:10.1016/j.diff.2010.12.003] [PMID]

Faroni, A., Mobasseri, S. A., Kingham, P. J., \& Reid, A. J. (2015) Peripheral nerve regeneration: Experimental strategies and future perspectives. Advanced Drug Delivery Reviews, 82-83, 160-7. [DOI:10.1016/j.addr.2014.11.010] [PMID]

Feneley, M. R., Fawcett, J. W., \& Keynes, R. J. (1991). The role of Schwann cells in the regeneration of peripheral nerve axons through muscle basal lamina grafts. Experimental Neurology, 114(3), 275-85. [DOI:10.1016/0014-4886(91)90153-4] [PMID]

Hejazian, L. B., Esmaeilzade, B., Ghoroghi, F. M., Moradi, F., Hejazian, M. B., Aslani, A., et al. (2012). The role of biodegradable engineered nanofiber scaffolds seeded with hair follicle stem cells for tissue engineering. Iranian Biomedical Journal, 16(4), 193-201. [PMID] [PMCID]

Hoffman, R. M. (2006). The pluripotency of hair follicle stem cells. Cell Cycle, 5(3), 232-3. [DOI:10.4161/cc.5.3.2397] [PMID] 
Ide, C. (1996). Peripheral nerve regeneration. Neuroscience Research, 25(2), 101-21. [DOI:10.1016/0168-0102(96)01042-5]

Ishikawa, N., Suzuki, Y., Dezawa, M., Kataoka, K., Ohta, M., Cho, H., \& Ide, C. (2009). Peripheral nerve regeneration by transplantation of BMSC-derived Schwann cells as chitosan gel sponge scaffolds. Journal of Biomedical Materials Research Part A, 89(4), 1118-24. [DOI:10.1002/jbm.a.32389] [PMID]

Lee, A. C., Vivian, M. Y., Lowe III, J. B., Brenner, M. J., Hunter, D. A., Mackinnon, S. E., et al. (2003). Controlled release of nerve growth factor enhances sciatic nerve regeneration. Experimental Neurology, 184(1), 295-303. [DOI:10.1016/S00144886(03)00258-9]

Li, Y., Guo, L., Ahn, H. S., Kim, M. H., \& Kim, S. W. (2014) Amniotic mesenchymal stem cells display neurovascular tropism and aid in the recovery of injured peripheral nerves. Journal of Cellular and Molecular Medicine, 18(6), 1028-34. [DOI:10.1111/jcmm.12249] [PMID] [PMCID]

Lin, H., Liu, F., Zhang, C., Zhang, Z., Guo, J., Ren, C., \& Kong, Z. (2009). Pluripotent hair follicle neural crest stem-cell-derived neurons and schwann cells functionally repair sciatic nerves in rats. Molecular Neurobiology, 40(3), 216-23. [DOI:10.1007/ s12035-009-8082-z] [PMID]

Matsumoto, K., Ohnishi, K., Kiyotani, T., Sekine, T., Ueda, H., Nakamura, T., et al. (2000). Peripheral nerve regeneration across an 80-mm gap bridged by a polyglycolic acid (PGA)collagen tube filled with laminin-coated collagen fibers: A histological and electrophysiological evaluation of regenerated nerves. Brain Research, 868(2), 315-28. [DOI:10.1016/S00068993(00)02207-1]

Millesi, H. (1984). Nerve grafting. Clinics in Plastic Surgery, 11(1), 105-13. [DOI:10.1016/S0094-1298(20)31826-5]

Mimura, T., Dezawa, M., Kanno, H., Sawada, H., \& Yamamoto, I. (2004). Peripheral nerve regeneration by transplantation of bone marrow stromal cell-derived Schwann cells in adult rats. Journal of Neurosurgery, 101(5), 806-12. [DOI:10.3171/ jns.2004.101.5.0806] [PMID]

Moradi, F., Bahktiari, M., Joghataei, M. T., Nobakht, M., Soleimani, M., Hasanzadeh, G., et al. (2012). BD PuraMatrix peptide hydrogel as a culture system for human fetal Schwann cells in spinal cord regeneration. Journal of Neuroscience Research, 90(12), 2335-48. [DOI:10.1002/jnr.23120] [PMID]

Nobakht, M., Hoseini, S. M., Mortazavi, P., Sohrabi, I., Esmailzade, B., Roosh, N. R., et al. (2011). Neuropathological changes in brain cortex and hippocampus in a rat model of Alzheimer's disease. Iranian Biomedical Journal, 15(1-2), 51-8. [PMID] [PMCID]

Quan, R., Zheng, X., Ni, Y., Xie, S., \& Li, C. (2016). Culture and characterization of rat hair follicle stem cells. Cytotechnology, 68(4), 621-8. [DOI:10.1007/s10616-014-9807-z] [PMID] [PMCID]

Rodríguez, F. J., Verdú, E., Ceballos, D., \& Navarro, X. (2000). Nerve guides seeded with autologous Schwann cells improve nerve regeneration. Experimental Neurology, 161(2), 571-84. [DOI:10.1006/exnr.1999.7315] [PMID]

Sieber-Blum, M., \& Grim, M. (2004). The adult hair follicle: Cradle for pluripotent neural crest stem cells. Birth Defects Research Part C: Embryo Today: Reviews, 72(2), 162-72. [DOI:10.1002/bdrc.20008] [PMID]
Sieber-Blum, M., Grim, M., Hu, Y. F., \& Szeder, V. (2004) Pluripotent neural crest stem cells in the adult hair follicle. Developmental DYNAMICS, 231(2), 258-69. [DOI:10.1002/ dvdy.20129] [PMID]

Walsh, S., Biernaskie, J., Kemp, S. W. P., \& Midha, R. (2009) Supplementation of acellular nerve grafts with skin derived precursor cells promotes peripheral nerve regeneration. Neuroscience, 164(3), 1097-107. [DOI:10.1016/j.neuroscience.2009.08.072] [PMID]

Wang, X., Hu, W., Cao, Y., Yao, J., Wu, J., \& Gu, X. (2005). Dog sciatic nerve regeneration across a 30-mm defect bridged by a chitosan/PGA artificial nerve graft. Brain, 128(8), 1897-910. [DOI:10.1093/brain/awh517] [PMID]

Yang, F., Murugan, R., Wang, S., \& Ramakrishna, S. (2005). Electrospinning of nano/micro scale poly (L-lactic acid) aligned fibers and their potential in neural tissue engineering. Biomaterials, 26(15), 2603-10. [DOI:10.1016/j.biomaterials.2004.06.051] [PMID] 
This Page Intentionally Left Blank 